\title{
Optimising the Performances of Hollow Antiresonant Fibres
}

\author{
F. Poletti, J. R. Hayes, D. J. Richardson \\ Optoelectronics Research Centre, University of Southampton, Southampton SO17 1BJ, United Kingdom \\ frap@orc.soton.ac.uk
}

\begin{abstract}
We study the loss of novel antiresonant hollow-core fibres, demonstrate the existence of a wavelength independent optimum distance between core-surround and solid outer cladding, and provide useful guidelines for the fabrication of practical low-loss fibres.

OCIS codes: (060.2280) Fiber design and fabrication; (060.4005) Microstructured fibers;
\end{abstract}

\section{Introduction}

Low loss hollow core fibres have revolutionised the field of gas-based nonlinear optics [1] and are set to play an increasingly important role in the delivery of extremely intense optical pulses. Hollow core photonic bandgap fibres (PBGFs) have been already employed for the delivery of a few $\mu \mathrm{J}$ for fs pulses [2] or $0.5 \mathrm{~mJ}$ for ns pulses [3]. For pulse energies exceeding the $\mathrm{mJ}$ level though, air cores larger than the 10-20 microns typically possible with these fibres are required to prevent damage in the dielectric material. For these applications, another class of hollow core fibres with a periodic arrangement of air holes in the cladding, separated by ultrathin membranes typically forming a Kagome' or square lattice, can provide the required 30-80 $\mu \mathrm{m}$ core diameters with sub-dB/m losses [4].

The lack of periodically arranged nodes in these fibres, and therefore the absence of a true photonic bandgap, has stimulated substantial research in recent years to understand their guidance mechanism and loss scaling rules. In an attempt to reduce the fibre complexity and thus isolate the structural features responsible for optical guidance, a few authors have recently looked at simpler fibre designs where the cladding consists of a single ring of holes around the central core [5-7]. Fevrier et al. have suggested that antiresonance alone from the glass tubular core surround may explain the guidance and account for the losses in these fibres. Based on this concept, they have proposed a simplified fibre design, consisting of a thin hexagonal core surround, supported by 6 thin membranes [5].

In this work we provide enhanced understanding of the guiding mechanism in these relatively simpler to make but potentially extremely useful antiresonant fibres. We argue that antiresonance from the annular core surround alone cannot explain the low losses experimentally achieved. Fresnel reflections from the outer solid jacket are found to play an important role too, and optimizing the distance $t$ between the external solid cladding and the annular core surround is essential to minimise losses. We derive an analytical formula expressing the optimum separation $t$ as a function of core radius and show the wavelength independence of this geometrical rule. We also study the effect that different core and supporting strut shapes have on the overall loss and show that a Star-of-David structure can have even lower losses than the optimum circularly symmetric fibre throughout most of the spectrum.

\section{Circularly symmetric fibre and the influence of the outer solid cladding}

We begin our study from the simplest (yet unfeasible) air core fibres possible, the circular air-glass Bragg fibres which can be conveniently studied using a simple semianalytic model based on a transfer matrix formulation [8]. As shown in Fig. 1(a), the well-known $\lambda^{2} / \mathrm{r}^{3}$ loss dependence in a simple circular hollow fibre results in impractically large losses, unless $r>\lambda$, for which the fibre becomes a highly multimoded and unbendable structure [9]. A tubular fibre with the same core diameter but only a thin glass annular surround has a $\lambda^{3} / \mathrm{r}^{4}$ loss dependence [10], hence lower losses for the same $\lambda / \mathrm{r}$. However, a lower loss level in most of the spectrum comes at a price of some localised, frequency-periodic high loss peaks, corresponding to radial resonances in the tubular surround. In this work we have found that this waveguide alone would not be able to account for the losses experimentally observed in the antiresonant fibre reported in [5]. Only when the presence of a solid outer glass cladding separated by a distance $t$ from the tube is also included and the Fresnel reflections at this additional glass-air interface are properly account for, do the calculated losses come close to the measured ones. Numerically we have found that the loss of this structure follows a $\lambda^{4} / \mathrm{r}^{5}$ dependence, Fig. 1(b), and that the distance $t$ significantly influences the overall loss, Fig. 1(a) and Fig. 2(a).

Bragg fibre's theory suggests that minimum loss can be achieved by imposing the quarter wave stack condition (QWSC) on each individual layer. By imposing the QWSC and by using a semianalytical formula for the fundamental mode effective index [9], we found that the optimum air thickness is, to a first order approximation:

$$
t_{\text {opt }}=\frac{\lambda}{4 \sqrt{1-n e f f^{2}}} \simeq \frac{\pi}{2 u} r \cong 0.65 r
$$

where $u$ is the first zero of the $\mathrm{J}_{0}$ Bessel function and $r$ the core radius. 
(a)

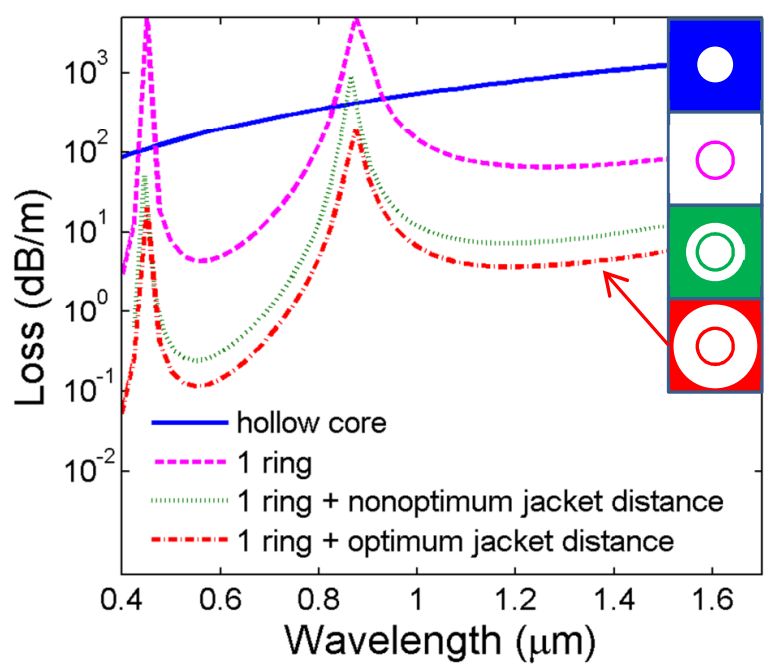

(b)

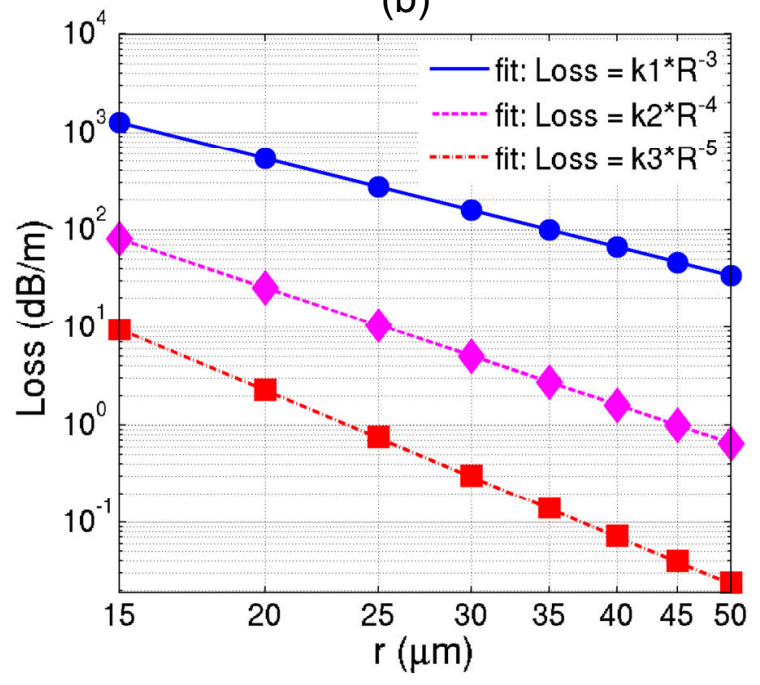

Fig. 1. (a) Calculated confinement loss of circularly symmetric glass-air structures: hollow core, tube in air and tube in a larger solid cladding with optimised and non-optimised distance. The curves refer to the fibres shown in the inset, where air regions are shown in white, the core radius is $\mathrm{r}=15 \mu \mathrm{m}$ and core surround thickness is $420 \mathrm{~nm}$. (b) Calculated loss vs radius $r$ for hollow core (circles), tube in air (diamonds) and tube in a larger cladding (squares).

It is worth noting that to a first order approximation the wavelength dependence of numerator and denominator cancel out, and for this particular fibre the optimum condition in (1) becomes wavelength independent. Therefore, if the structure satisfies (1) its losses will reach the minimum possibly achievable value at all wavelengths, as shown in Fig. 1(a). Fig. 2(a) illustrates the sensitivity of the loss to the ratio $t / r$ and shows that for normalised thicknesses of the annular air clad region in the 0.6-0.8 range the loss is close to the minimum value, but for thinner or thicker air regions the loss can easily worsen by an order of magnitude or more. This clearly demonstrates the need to carefully take the outer air clad into consideration when designing more realistic fibres.

\section{Realistic cladding designs}

Having understood important design rules for the perfectly circular core case, we now turn our attention to more realistic designs. In any real air-glass antiresonant fibre, the core surround must be physically interconnected to the outer glass cladding. By matching the thickness of the interconnecting struts to that of the core surround one can avoid the appearance of additional large resonant peaks in the spectrum. However this also severely decreases the symmetry class of the fibre from the $\mathrm{C}_{\infty \mathrm{ov}}$ of the circular case. As a result, undesirable additional resonant peaks corresponding to azimuthal resonances appear in the spectrum (e.g. compare curves iii and iv in Fig. 2(b)).

(a)

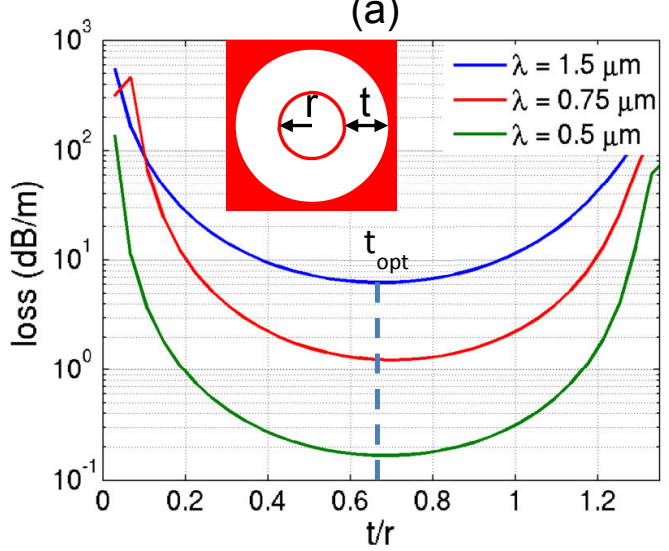

(b)

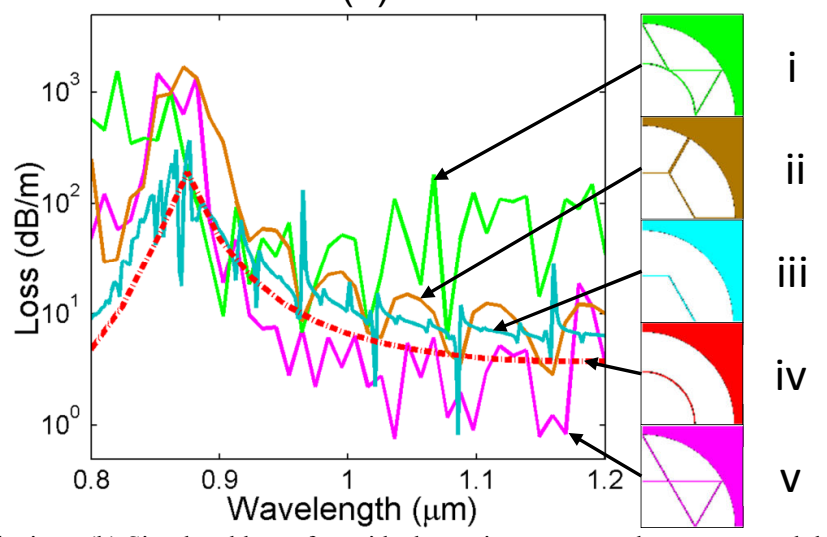

Fig. 2. (a) Loss versus normalised thickness for the structure in the inset (b) Simulated loss of two ideal, non-interconnected structures and three realistic fibres. For all the core radius is $r=15 \mu \mathrm{m}$ and core surround thickness is $420 \mathrm{~nm}$. 
These minor loss peaks correspond to avoided crossings between the air guided mode and the multitude of leaky whispering-gallery-like modes in the core surround belonging to the same symmetry class. Such spurious additional resonances cannot be avoided in any real structure, even though asymmetric core shapes or n-sided polygons with a large number of sides are expected to mitigate their spectral occurrence.

We finally moved on to study the loss of several anti-resonant fibres with the same core size but different core shapes and interconnections. For this task we used a finite element method (FEM) modal solver, previously calibrated using the Bragg fibre results. A selection of some of the fibres considered is shown in Fig. 2(b). As expected, most geometries generally have higher loss than the ideal tubular fibre with an optimum outer cladding (curve iv). However, the Star of David fibre design (curve v) is found to have lower losses throughout most of the antiresonant window. This is surprising, considering that the core size is the same as in fibre iii and that both correspond to a suboptimum value of $\mathrm{t} / \mathrm{r}=1$. In order to investigate these findings experimentally, we have fabricated the two different antiresonant fibres shown in Fig. 3. The presence of small nodes in the real fibres was found to increase the loss considerably [11] and to make any fair comparison between different structures very hard. Work is currently ongoing to further reduce the node sizes and understand the origin of the low loss in structure $\mathrm{v}$.

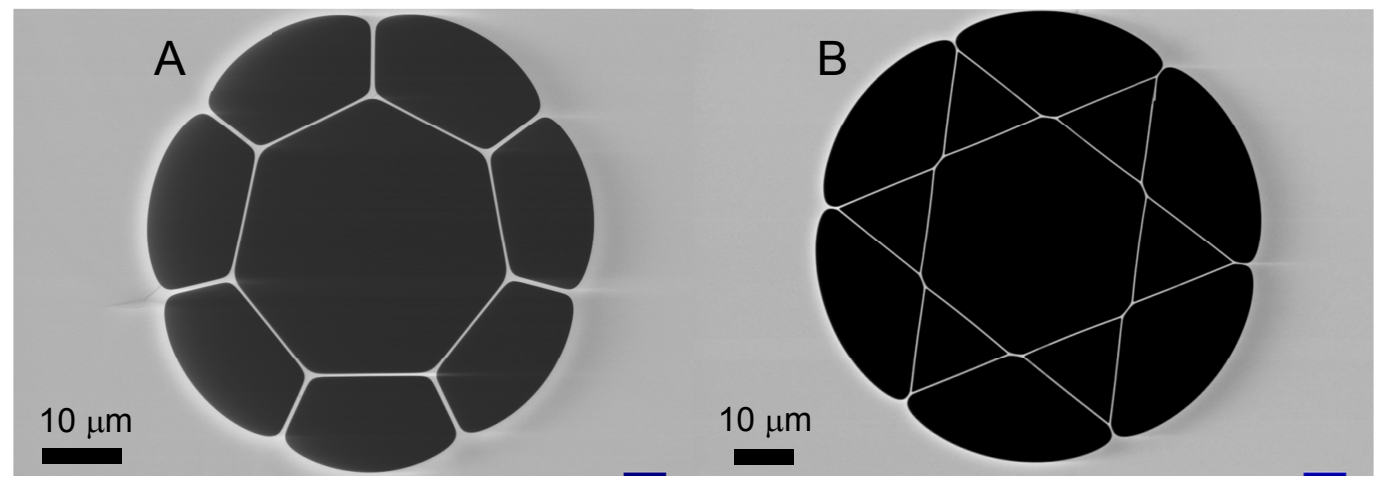

Fig. 3. SEM of two fabricated antiresonant hollow core fibres with: $(A) r=32.8 \mu \mathrm{m}$, surround thickness of $340 \mathrm{~nm}$ and $\mathrm{t} / \mathrm{r}=0.75 ;(\mathrm{B}) \mathrm{r}=38.6$ $\mu \mathrm{m}$, surround thickness of $300 \mathrm{~nm}$ and $\mathrm{t} / \mathrm{r}=1.1$. Despite a more favourable $\mathrm{t} / \mathrm{r}$, due to its larger nodes, fibre $\mathrm{A}$ is found to have higher losses.

\section{Conclusions}

We have studied loss mechanisms and scaling rules of a novel class of antiresonant hollow core fibres, and shown that the presence of an outer glass cladding is essential, not only to add structural rigidity, but also to reduce the fibre losses. In these fibres, the minimum loss is achieved for a cladding distance close to the optimum $t \sim 0.65 x r$, which satisfies the quarter wave stack condition and is found to be wavelength independent. Furthermore, by virtue of a favourable $\lambda^{4} / \mathrm{r}^{5}$ loss dependence as compared to the $\lambda^{2} / \mathrm{r}^{3}$ of more conventional circular hollow core fibres, the same loss can be achieved with smaller core sizes, Fig. 1(b), with obvious advantages in terms of lower intermodal crosstalk and bending losses. The numerical study of various realistic core shapes indicates that even apparently similar fibres can have loss differences of nearly two orders of magnitude (e.g. i and v in Fig.2(b)). Amongst all the realistic structures investigated, the Star-of-David fibre is found to exhibit the lowest losses. We believe that the design rules described in this paper will prove important in driving the realisation of simple and novel low-loss, large core, air guiding fibres. We gratefully acknowledge support from the Engineering and Physical Sciences Research Council in the UK for funding of this work under the EPSRC Centre for Innovative Manufacturing in Photonics.

\section{References}

[1] F. Benabid et al., "Stimulated Raman Scattering in Hydrogen-Filled Hollow-Core Photonic Crystal Fiber," Science 298, 399-402 (2002).

[2] C. J. Hensley et al., "Extremely High Coupling and Transmission of High-Powered-Femtosecond Pulses in Hollow-Core PhotonicBandGap Fiber," CLEO 2008, paper JFG1

[3] J. D. Shephard et al., "Improved hollow-core photonic crystal fiber design for delivery of nanosecond pulses in laser micromachining applications," Appl. Opt. 44, 4562 (2005).

[4] F. Couny et al., "Generation and Photonic Guidance of Multi-Octave Optical-Frequency Combs," Science 318, 1118-1121 (2007).

[5] S. Février et al., "Understanding the origin of loss in large pitch hollow-core photonic crystal fibers," Opt. Express 18, 5142-5150 (2010).

[6] A. D. Pryamikov et al., "Demonstration of a waveguide regime for a silica hollow-core microstructured optical fiber with a negative curvature of the core boundary in the spectral region > $3.5 \mu \mathrm{m}$," Opt. Express 19, 1441-1448 (2011).

[7] Luca Vincetti and Valerio Setti, "Waveguiding mechanism in tube lattice fibers," Opt. Express 18, 23133-23146 (2010).

[8] Pochi Yeh, Amnon Yariv, and Emanuel Marom, "Theory of Bragg fiber," J. Opt. Soc. Am. 68, 1196-1201 (1978).

[9] E. Marcatili and R. Schmeltzer, Bell Syst. Tech. J. 43, 1783-1809 (1964).

[10] M. Miyagi, "Bending losses in hollow and dielectric tube leaky waveguides," Appl. Opt. 20, 1221-1229 (1981).

[11] J. R. Hayes et al.,"Reducing loss in practical single ring antiresonant hollow core fibres," CLEO EUROPE 2011, paper CJ2.2 